Mots. Les langages du politique

De la vitupération. Le pamphlet et les régimes du « dire vrai » en politique

Michel Hastings

\title{
CpenEdition
}

Journals

Édition électronique

URL : https://journals.openedition.org/mots/19188

DOI : $10.4000 /$ mots. 19188

ISSN : 1960-6001

Éditeur

ENS Éditions

Édition imprimée

Date de publication : 30 novembre 2009

Pagination : 35-49

ISBN : 9782847881820

ISSN : 0243-6450

Référence électronique

Michel Hastings, «De la vitupération. Le pamphlet et les régimes du « dire vrai » en politique », Mots. Les langages du politique [En ligne], 91 | 2009, mis en ligne le 30 novembre 2011, consulté le 23 avril 2022. URL : http://journals.openedition.org/mots/19188; DOI : https://doi.org/10.4000/mots.19188 


\section{De la vitupération. Le pamphlet et les régimes du «dire vrai » en politique}

Profaner c'est restituer à l'usage commun ce qui a été séparé dans la sphère du sacré. (Agamben, 2005)

«Le pamphlet devrait être la forme littéraire par excellence d'une époque comme la nôtre. Nous vivons un temps où les passions politiques sont vives, où les canaux d'une expression libre se raréfient et où le mensonge organisé existe à une échelle encore jamais vue. Pour remplir les blancs de l'histoire, le pamphlet est la forme idéale» (Orwell, 1943, p. 356). George Orwell assignait au pamphlet une double propriété : incarner, tout d'abord, l'historicité singulière d'un mode d'écriture, lier un genre littéraire à l'expérience particulière d'une époque; combler, ensuite, les béances d'une période de crise en mettant le logos pamphlétaire au service de la liberté et de la vérité. Nous retrouvons cette idée d'un «pamphlet symptomatique » chez Marc Angenot pour qui ce type de discours agonique, pertinent dans une certaine société, accompagnerait «dans le champ social de brusques ruptures entre un ensemble de valeurs et des pratiques concrètes» (Angenot, 2005, p. 39). Voilà qui semble lier la forme pamphlétaire à un moment précis, défini par l'épaisseur des contradictions idéologiques, les conflits d'idées et la complexité des rapports aux pouvoirs symboliques de la part de certains agents sociaux. Pour le dire autrement, le pamphlet serait donc mortel.

Notre propos n'est pas de signer une fois de plus l'acte de décès du pamphlet, ni d'ailleurs de convaincre de son indémodable permanence, mais de formuler un certain nombre d'hypothèses relatives à la généalogie d'une parole vitupérative, que nous entendons définir principalement autour de deux éléments. Tout d'abord, un mode d'énonciation publique du blâme à l'encontre d'une autorité/institution politique ou sociale. Il s'agit donc ici de repérer le montage de la vitupération comme agencement de contraintes discursives, de conditions de mise en publicité et de finalités critiques. Ensuite et surtout, une 
appartenance au répertoire plus général des «dramatiques de la vérité» dont Michel Foucault avait commencé à entreprendre la vaste archéologie. La vitupération dessine, selon nous, les contours plus ou moins flous d'un espace public de la critique, dont il ne faut certes pas exagérer le degré d'institutionnalisation, mais qui témoigne néanmoins, dans le cadre de configurations précises, de manières particulières de dire vrai, ou, pour reprendre le lexique de la pragmatique linguistique, un « je vrai » qui revendique le statut du « on vrai » (Berrendonner, 1981). Dès lors, le pamphlétaire serait la figure sociohistorique d'une formule plus générique, une manière parmi d'autres de témoigner du scandale de la vérité. Par son écriture polémique et son franc-parler, par ses accents indignés et ses poses héroïques, il entrerait, certes sous une forme bien altérée, dans la grande famille de ceux qui par le verbe et le corps ont illustré, ou cru illustrer, «le courage de la vérité » (Foucault, 2008, 2009).

Ce jeu d'hypothèses va nous permettre d'organiser notre réflexion autour de trois séries de questions. Est-il pertinent d'envisager l'inscription du pamphlet politique dans une mémoire longue du genre vitupératif? De quel âge d'or pamphlétaire serions-nous aujourd'hui orphelins? Comment se recompose, dans le cadre de la démocratie contemporaine et de ses actuelles mutations, un nouvel espace de la vitupération?

\section{Les régimes de la vitupération}

Vituperare : trouver des défauts, blâmer, réprimander, critiquer, faire le procès de; vituperator : censeur, détracteur, critique; vituperabilis : répréhensible, digne d'être critiqué. La notion de vituperatio renvoie à une activité extrêmement banale, la capacité de chaque individu d'émettre un jugement dépréciatif sur l'action ou la personnalité d'autrui. La dimension sociale et politique est également présente dans la définition. La vitupération repose en effet sur l'existence nécessaire de standards de justice au nom desquels se réfère plus ou moins consciemment le jugement (Tilly, 2008). Vitupérer entend rappeler une loi morale et marquer une distance entre celui qui blâme et celui qui est blâmé. La vitupération participe donc du processus de conflictualité des mondes qui traverse chaque société et qui voit chacune d'entre elles organiser un espace public du blâme au sein duquel la critique s'institutionnalisera dans des discours de la vérité qui revendiqueront au nom de celle-ci le pouvoir de tout dire (Boltanski, Thévenot, 1991).

\section{Vitupération et parrhésie}

L'éloquence romaine, au premier siècle avant notre ère, réservait une place importante à l'éloge des amis et à l'invective contre l'adversaire. Avec la lau- 
datio, la vituperatio constituait un registre discursif à part dans la rhétorique, le genre démonstratif ou épidictique. Elle venait illustrer la violence normale d'une éloquence où l'amplification était la règle, où le discours se voyait volontiers comparé à un feu, à un torrent, où il était conseillé de simuler les passions les plus fortes. La vitupération tire une grande partie de ses effets du langage judiciaire, notamment de l'impératif d'accusation qui construit la diatribe comme une indignation et une dénonciation. Elle fait de l'orateur le juge éloquent d'un scandale (Achard, 1981). Cicéron élèvera la vitupération au rang d'un art civique destiné à moraliser la cité et à dissuader les citoyens de rejoindre le camp des improbi. Il mobilisera un répertoire considérable de fondements à l'invective dont la plupart ont traversé les siècles pour constituer le jeu pamphlétaire de l'insulte et de la caricature (l'origine étrangère, l'immoralité, les traits physiques, l'incompétence et l'impuissance, les méfaits). La vitupération délivre un blâme public au nom d'un abandon de la morale en politique. Faiblement idéologique, elle apparait bien souvent comme une sorte de compensation à l'impossibilité d'agir, et met en scène une frustration que l'orateur s'efforce de conjurer en livrant alors le combat des mots.

La théâtralité de la vitupération romaine témoigne de l'existence et de la reconnaissance d'un espace discursif public livré au jeu des critiques, des assauts et des charges (Dupont, Valette-Cagnac, 2004). Foyer de l'éloquence, cet espace dessine également les contours d'une politique du franc-parler, avec en son cœur le geste de celui qui prend la parole en son nom propre. Michel Foucault a montré l'importance de la notion de parrêsia (de deux mots grecs, pan «tout » et rhema "ce qui est dit»): d'abord, dans le cadre des vertus et des techniques que l'on doit rencontrer chez celui qui dirige la conscience des autres et les aide à constituer leur rapport à soi (Foucault, 2001); ensuite, dans le champ politique, où le concept désigne une certaine manière de dire la vérité, une manière qui ne porte pas sur la structure interne du discours, ni sur ses finalités visées, mais qui est à rechercher du côté de celui qui parle, et plus précisément du risque que le dire vrai ouvre pour le locuteur lui-même. «Il y a parrêsia lorsque le dire vrai se dit dans des conditions telles que le fait de dire la vérité, et le fait de l'avoir dite va ou peut ou doit entraîner des conséquences coûteuses pour ceux qui ont dit la vérité.» (Foucault, 2008, p.46) En effet, dans la Grèce de Démosthène et des Philippiques, le parrèsiaste est celui qui donne son opinion, signant en quelque sorte lui-même la vérité qu'il énonce, mais cette vérité qu'il marque comme étant son opinion doit en plus affronter le risque de blesser l'autre, de l'irriter. L'espace public discursif dont nous recherchons ici la généalogie est donc un espace de risque. La parole de vérité ouvre un danger et celui qui parle le fait en connaissant l'existence du péril. La vitupération se ressource ainsi au cœur d'une dramaturgie du défi et de la liberté. 
Il y a parrêsia lorsqu'il y a liberté dans l'énonciation de la vérité, liberté de l'acte par lequel le sujet dit la vérité, et liberté aussi de pacte par lequel le sujet qui parle se lie à l'énoncé et à l'énonciation de la vérité. Et dans cette mesure-là, au cœur de la parrêsia, on ne trouve pas le statut institutionnel du sujet, on y trouve son courage. (Foucault, 2008, p.63)

Michel Foucault parlera même d'une sorte de contrat entre celui qui prend le risque de dire toute la vérité et celui qui accepte de recevoir comme vraie la vérité blessante qu'il entend (Foucault, 2009).

On peut formuler l'hypothèse selon laquelle la parrêsia constitue la matrice originelle des différentes expériences du dire vrai en politique, le creuset où se forgent les multiples expressions du «courage de la vérité », où se noue enfin la longue chaine des diverses figures de la critique publique qui ont toutes en commun de s'obliger à la vérité et de s'obliger par la vérité. Il était donc une fois une exigence de dire vrai, une prise de parole courageuse de vérité apparue chez les Grecs et qui connaitra au fil des configurations historiques de nombreux réinvestissements. On notera que la parrêsia ne se pense jamais comme un droit fondamental du citoyen, ni même comme une compétence technique à inclure dans l'art de gouverner des dirigeants. En revanche, elle semble s'enraciner dans l'expérience d'une tension agonistique qui voit l'orateur engager dans les affaires de la cité sa parole libre au nom d'une vérité irréductible. C'est parce que le parrèsiaste prend l'allure d'un homme qui se dresse en face d'un tyran et lui dit la vérité, qu'il inaugure, de façon paradigmatique, la longue histoire de ceux qui un jour ont incarné ou prétendu incarner le scandale du «tout dire».

\section{Le scandale du «tout dire»}

Michel Foucault, dans ses deux derniers cours au Collège de France, avait commencé à dessiner quelques figures historiques de cette subversion qui consiste non seulement à tout dire mais aussi à démasquer le non-dit et à passer outre la difficulté de dire : l'ascète chrétien, le révolutionnaire militant, l'artiste maudit. Il voyait en eux les fils héritiers des Cyniques qui avaient réussi à faire de leur forme de vie le lieu d'émergence même de la vérité. La vie d'artiste, celle du révolutionnaire ou du moine ont en commun de reposer sur deux principes : l'art, l'idéologie ou la foi sont, tout d'abord, capables de donner à l'existence une forme en rupture avec toute autre, une expérience ontologique de la limite comme l'ont éprouvée le nihiliste, le maudit ou le franciscain; et ensuite, l'idée selon laquelle la pratique de l'art, de l'idéologie ou de la foi constitue une expérience de mise à nu de soi, de retour à l'authentique, comme si la vérité témoignait à travers la vie même de ces acteurs. Bien entendu, la liste des descendants du parrèsiaste n'est pas close, et nous souhaitons l'entrouvrir à la grande famille des vitupérateurs. À tous ceux qui ont 
pris la parole, par le verbe ou l'écriture, et ont un jour clamé publiquement leur indignation en dénonçant, ici, le despote déguisé en monarque, là, le prince corrompu, ailleurs, la trahison des élites. À tous ceux qui, libellistes, satiristes, caricaturistes, libertins, pamphlétaires et autres polémistes, ont, au fil des siècles, rejoué la scène primitive de la parrhésie, en indexant parfois leur vie au pouvoir revendiqué de tout dire. Prenons deux exemples.

Soit, tout d'abord, les mazarinades, c'est-à-dire cet ensemble hétéroclite de textes, chansons, récits, placards, publiés lors des temps troublés de la Fronde, et qui témoignent paradoxalement, par leur écriture libérée et débridée, des impasses de la pensée critique face à l'absence d'alternative politique. La tonitruance du verbe fonctionne alors bien souvent comme un trompe-l'œil, comme l'aveu d'un échec à pouvoir faire changer les choses. Cette littérature, dont les auteurs recherchaient le plus souvent l'anonymat pour se protéger des risques de châtiment, est destinée à circuler et donc à dessiner les contours d'un espace public où les opinions s'affrontent au gré des évènements. Les mazarinades sont profondément périssables, éphémères, portées par les rumeurs et les faits divers, mais ce corpus, qui se prête si peu aux études sérielles, riche d'insultes et d'imprécations, met en scène, comme l'a très bien démontré Christian Jouhaud, une sorte de radicalisme de l'impuissance et de l'ostentation (Jouhaud, 1985). Le dire vrai s'épuise dans un excès de virulence et se met au service de la tactique. Le «courage de la vérité » est certes ici totalement happé par la dynamique des coups qu'il convient de porter à l'adversaire, mais il inaugure un vaste et double mouvement de politisation de la littérature et de littérarisation du politique, qui permettra, au $18^{\mathrm{e}}$ siècle, à la littérature de devenir à la fois un refuge critique et un tribunal moral (Koselleck, 1979; Jouhaud, 2000), et à l'écrivain d'entamer dans l'ombre contraignante des Académies sa progressive montée en singularité. Les auteurs de ces textes, empêtrés dans leurs ruses discursives et leurs jeux de la faux-semblance, n'ont pas l'allure flamboyante des héros de la vérité, et pourtant ils l'incarnent, à leur manière et dans le cadre sociohistorique complexe de structures d'opportunité réduites. Ils blâment Mazarin, vitupèrent contre sa clique, prennent des risques au nom d'un dire vrai réduit à l'horizon de leur faction. Ils sont les figures appauvries d'une parrêsia, certes abâtardie, mais qui fonctionne néanmoins comme un référentiel lointain prêt à alimenter de nouveaux avatars.

Soit, ensuite, Donatien-Alphonse-François de Sade qui, à l'ombre des Bastilles, aura le temps de méditer sur ce qu'il en coute non seulement d'outrager la loi, mais aussi de s'entêter dans l'irrédemption (Ost, 2005, p. 10). Au cœur du dispositif sadien, il y a «une écriture sans merci» dont l'entière logique consiste à tout dire et notamment là où se construisent les frontières sociales de l'indicible et de l'innommable. Le tout dire de Sade est le paroxysme d'une violence d'autant plus forte qu'elle est simple. Une parole sans équivoque, 
sans métaphore, privée de toute arrière-pensée et qui n'épargne rien. La subversion conduit Sade à rechercher la vérité là où elle se tait, à l'écrire avec outrance quand elle préfère se cacher, à la trouver dans la nature plutôt que dans la loi : «Le motif qui m'engage à me livrer au mal est né chez moi de la profonde étude que j'ai faite de la nature. Plus j'ai cherché à surprendre ses secrets, plus je l'ai vue uniquement occupée de nuire aux hommes. » ( $L a$ Nouvelle Justine) Le dire vrai chez Sade est tout entier porté par un idéal de révélation et sa vitupération trouve dans le blasphème la seule catégorie à ses yeux acceptable de véridiction. La parole transgressive dévoile la vérité et c'est par la profanation langagière que le libertin accède à l'immoralité institutrice. Sade ne cesse de corrompre l'autorité de la loi en la poussant aux limites de ses contradictions : "J'ose assurer, en un mot, que l'inceste devrait être la loi de tout gouvernement dont la fraternité fait la base.» (Français, encore un effort si vous voulez être républicains) La Révolution ne va décidément pas jusqu'au bout de ses idéaux! Sade entend donc faire éclater une vérité en se lançant à l'assaut des lois sociales dont il entend dénoncer les crimes, les mensonges et les impostures, faire éclater la vérité jusqu'à l'emmurement de sa vie. Ne souscrit-il pas lui aussi au pacte originel de la parrhésie?

Tel un filigrane, l'esprit de la parrêsia semble donc parcourir les siècles. Chaque époque construit son dire-vrai, son scandale de la vérité et ses témoins indignés. Chaque époque possède ses vitupérateurs, son répertoire du blâme et ses irrépressibles besoins de tout dire. Bien entendu, au regard du modèle originel de la Grèce antique, les héritages apparaitront parfois bien altérés, dégradés, les héritiers donneront l'impression souvent désagréable de jouer "petits bras» et d'investir des vérités à leur mesure, médiocres. L'essentiel ne réside cependant pas, selon nous, dans la comparaison avec un quelconque prototype. L'intérêt de construire une généalogie de la parrhésie consiste à faire apparaitre une structure élémentaire des sociétés politiques : la présence d'un lieu critique d'où émerge une collection de figures historiques de la vitupération. Le pamphlétaire en est une, tout aussi passagère que les autres, mais également tout aussi exemplaire.

\section{L’âge d’or du projet pamphlétaire}

L'hypothèse de l'actuelle disparition des pamphlets politiques se fonde en partie sur la référence à un âge d'or. La vitupération pamphlétaire aurait ainsi connu en France ses riches heures entre 1850 et 1940 , un siècle au cours duquel la littérature de type agonique se développa, enrichissant le discours social d'une parole polémique et violente, assurant la gloire de quelques bretteurs avides de scandales à dénoncer, consacrant enfin le règne d'un champ de la critique à la croisée des mondes journalistique, politique et littéraire. 
Revenir sur le pamphlet, c'est non seulement comprendre de quoi nous sommes aujourd'hui privés, mais c'est aussi prendre conscience des mutations profondes qui ont remodelé le paysage de la vitupération.

\section{L'opération pamphlétaire}

Nous appellerons «opération pamphlétaire » une activité à la fois discursive, sociale et politique qui ne se réduit pas à l'énonciation d'un texte a fortiori fugace et bien souvent anecdotique, mais qui entend au contraire restituer les dynamiques internes et les enjeux d'un «monde» du pamphlet que nous structurons ici autour de trois principaux éléments.

L'opération pamphlétaire se caractérise, tout d'abord, par un travail d'écriture. Parole aisément reconnaissable dont Marc Angenot a très bien analysé les invariants lexicaux et les trucs rhétoriques (Angenot, 2005) mais dont il exagère quelque peu l'originalité. Il nous semble en effet que tant les lexiques que les effets de la parole pamphlétaire ressortissent à une famille vitupérative beaucoup plus ancienne, aux origines composites. Si l'on a rapproché l'écriture des pamphlets antisémites de l'entre-deux guerres d'une matrice (contre-) révolutionnaire illustrée notamment par les libelles de Marat, Hébert et du Père Duchesne (Roussin, 2005), il conviendrait également de souligner les emprunts de la rhétorique de l'invective aux techniques de la prédication misant sur les effets les plus pathétiques, ainsi qu'aux langages mondains de la critique développés dans certains segments sociaux aristocratiques (Chartier, 2005).

Un pamphlet, c'est peut-être ensuite et surtout un pamphlétaire. C'està-dire un personnage qui inscrit son être et son agir dans un «registre de la singularité», et qui revendique un ethos fait d'irréductibilité, d'héroïsme, de solitude et d'indignation. Il est le réfractaire qui défie les autorités et dont le franc-parler fait trembler les puissants. En ce sens, le pamphlétaire épouse le processus plus général de singularisation qui voit au $19^{e}$ siècle l'éthique artistique mobiliser le régime vocationnel, miser sur la croyance en sa propre élection pour conforter son idéal d'autonomie. Une anthropologie de l'admiration permettrait probablement de comprendre la manière dont s'est constituée à l'époque la gloire, puis l'oubli du pamphlétaire (Heinich, 1991). Cette posture qui consiste à juxtaposer l'ego et l'universel, sans aucune médiation, sans jamais parler au nom d'une quelconque collectivité, cette façon de sermonner au nom d'une morale venue du for intérieur, ne fait-elle pas du pamphlétaire une "paraphrase», pour parler comme Clifford Geertz, une figure sécularisée de la prédication et du prophète wébérien? En ce sens, le pamphlétaire incarnerait celui qui peut, «à la différence du porte-parole institutionnel, représenter les autres sans mandat, qui tirerait argument de son indépendance, de sa solitude, de sa position hors la cité, pour réclamer d'être suivi et écouté» (Boltanski, 1985, p. 31). 
Le projet pamphlétaire ne saurait enfin se confondre avec l'CFuvre du Grand Homme, sauf à faire abstraction de l'ensemble des conditions et déterminations qui rendent possible et pensable toute cette opération. Il existe au $19^{\text {e }}$ siècle une condition pamphlétaire, ce qui permet de ne plus considérer le pamphlet comme un acte isolé, mais inséré dans le réseau de ses interdépendances, dans les cadres pratiques de sa création. Ainsi définie, l'opération pamphlétaire montre une forme de vitupération profondément redevable d'un ordre politique, social et culturel marqué tout d'abord par l'affirmation des processus de démocratisation, de politisation et de nationalisation qui affectent la compétition politique (Joanna, 1999), également par les mutations touchant à la définition du rôle de l'écrivain comme subjectivité triomphante, par le développement enfin de la presse populaire, lui-même soumis à une forte circularité entre les formes littéraires et les formes journalistiques (Thérenty, 2007).

La vitupération pamphlétaire se déploie donc dans un nouvel espace public du tout dire où le rôle de «conscience observante » singularise la parole politique, héroïse l'indignation et prophétise l'horizon national. Le pamphlétaire se transforme en chef d'orchestre d'un discours social et politique qui s'enivre de ses premières libertés. Il distribue les blâmes et fustige les mœurs politiques. Mais ses insolences le conduisent très souvent à se reposer sur des rituels de vitupération, à jouer l'acteur d'un théâtre politique bien huilé. On peut donc faire l'hypothèse d'une parrhésie tribunitienne qui, loin de déstabiliser les autorités, aurait plutôt eu pour effet de les conforter, en verbalisant de manière routinière les indignations, en les canalisant vers des dénonciations de plume, en permettant aux institutions judiciaires et politiques d'ajuster les frontières de l'acceptable et de l'interdit. On illustrera cette hypothèse en liant le pamphlet à l'histoire sociale de la colère.

\section{Le thymos pamphlétaire}

La forme pamphlétaire de la vitupération, ses succès et ses limites, peuvent en effet s'analyser à la lumière du projet de l'époque, consistant à construire l'affectivité révolutionnaire autour du sentiment de colère. Vers la colère doit confluer toute la misère des humiliés - c'est ainsi que le militantisme du 19 e siècle entendait inscrire son action. Comme l'a très bien montré Peter Sloterdijk,

parce que la société souffre avant tout d'un manque impardonnable de colère manifeste contre sa propre situation, développer une culture de l'indignation en encourageant méthodiquement la colère devient la principale mission psychopolitique de l'époque qui commence au cours de la Révolution française. (Sloterdijk, 2007, p. 164) 
Une grande partie des pratiques militantes devient impensable sans la mobilisation d'une rhétorique thymotique (thymos prend ici le sens d' «élément ardent de l'âme » ouvrant le terme à toutes les variations autour de la "bile noire », de la colère, du courage et de la gloire). La colère exprime le sentiment du siècle, elle envahit progressivement l'ensemble du discours social et les entrepreneurs de colère se dressent en figures rassembleuses autour de projets où la violence, la guerre civile, la révolution s'énoncent comme autant de réponses aux justes colères du peuple. L'imaginaire littéraire mais également les sciences sociales naissantes (notamment l'anthropologie criminelle) construisent leurs préjugés et leurs représentations du peuple autour du spectre d'une foule furieuse, ivre et dangereuse, dont les terribles colères deviendront les sources de récits hallucinants (Barrows, 1990). Taine, Zola, Sighele et Le Bon communient tous autour du même fantasme : la dangerosité des foules. «Et c'était sous l'air glacial, une furie de visages, des yeux luisants, des bouches ouvertes, tout un rut de peuple... » (Germinal). La colère alimente les angoisses bourgeoises, elle définit aussi la geste gréviste, transformant «l'échappée belle des usines » en de formidables rêves d'incendies et de Grand Soir. Les masses ouvrières se socialisent et se conscientisent comme classe au travers de ce pathos colérique. Au tournant du $19^{\mathrm{e}}$ siècle, la colère participe enfin du grand récit socialiste où se diffusent les topoi de la vengeance, de l'amertume et du soulèvement salutaire. La colère est saine car la cause est juste (Angenot, 1997).

La colère revêt donc une forme doxique et semble ponctuer l'ensemble des discours politiques et sociaux d'une époque où la neutralisation des violences physiques et verbales par la morale démocratique n'est pas encore achevée. C'est à cette culture thymotique qu'il convient de rapporter la vitupération pamphlétaire. C'est en effet bien souvent au nom d'une colère qu'il veut rendre publique que le pamphlétaire prend la plume, c'est elle également qui justifie et excuse les outrances verbales de l'assaut et de l'insulte (Bouchet, 2005; Chauvaud, 2008; Deleplace, 2009). L'irrigation du champ social et politique par la colère correspond probablement à une étape du processus de démocratisation, caractérisée par la faible institutionnalisation des organes de médiation. La posture colérique concentre sur elle un potentiel revendicatif au moment où les entreprises de représentation font encore défaut. Lorsque celles-ci se développeront, le statut de la colère changera. Peter Sloterdijk montre en effet comment les partis politiques et les syndicats ouvriers vont progressivement se transformer en «banques» nationales de la colère, la collectant et la travaillant afin de la soumettre aux impératifs nouveaux de l'idéologie. La colère des défavorisés fera ainsi l'objet d'un processus d'administration et de rationalisation. Ces organisations

donnent à la liaison entre les avoirs en colère et l'exigence de dignité une forme pragmatique, médiale et politique. La base de leur commerce est la promesse faite à leurs clients de déverser un profit thymotique sous forme d'une hausse du respect 
de soi et d'une capacité élargie à faire face à l'avenir s'ils renoncent au défoulement instantané de leur colère. (Sloterdijk, 2007, p.199)

On peut donc faire l'hypothèse que ce déplacement de la colère, et surtout son entrée dans le répertoire discursif et programmatique des partis politiques, entrainent une certaine civilisation des mœurs militantes. Le tout faire, mais aussi le tout dire feront progressivement l'objet de critiques. Le droit et la morale vont circonscrire les expressions hier encore légitimes de la colère. Le pamphlet n'est-il pas dès lors victime de ce processus d'euphémisation qui fait de la colère l'indice archaïque d'une culture populaire prédémocratique? Désormais, la colère va se trouver piégée par des dispositifs d'invocation parfaitement ritualisés (la fameuse «colère des travailleurs») mais aussi de plus en démonétisés. Certes, de grandes relances colériques auront lieu entre les deux guerres de la part notamment du Parti communiste (la grève de Douarnenez, les mobilisations contre le fascisme), et les pamphlets se mettront à proliférer à l'extrême droite pour ce que l'on peut considérer comme leur chant du cygne. Mais la haine s'est alors déjà depuis longtemps substituée à la colère.

En renouant ainsi les liens entre l'histoire sociale de la colère et la production pamphlétaire, nous avons essayé de rappeler que l'expérience de la vitupération s'inscrivait dans une tradition de «courage de la vérité» dont l'expression s'accompagne d'un risque pour l'énonciateur. L'indignation solitaire du parrèsiaste se convertit, avec le phénomène de démocratisation politique, en un sentiment de colère à faire partager. Le pamphlétaire est l'une des dernières figures de la révolte individuelle en un siècle qui inaugurera la massification des affects. La vérité commence à sortir du nombre.

\section{Un nouvel espace de la vitupération?}

La question semble entendue, le pamphlet serait mort. Le genre aurait disparu, les grandes figures de la plume acerbe auraient aujourd'hui rendu l'âme. On peut certes encore discuter de la date de décès ou du nom du dernier « véritable» pamphlétaire (Jean-Edern Hallier? Oriana Fallaci? Éric Zémour?), mais le constat est partout identique : il n'existerait plus aujourd'hui une production littéraire susceptible de prolonger le genre pamphlet. N'allons pas trop vite. D’une part, le pamphlet ne saurait-il pas déborder le champ littéraire? D'autre part, en faisant du pamphlet une formule sociohistorique particulière de la critique vitupérative, ne pourrait-on pas envisager l'hypothèse d'une recomposition de l'espace public de la vitupération? Où et comment aujourd'hui s'exprime le pouvoir de tout dire? Le dire vrai a-t-il trouvé ses nouveaux héros? Assisterions-nous à un nouvel âge de la parrhésie, tenant compte aussi bien des révolutions technologiques dans le monde des médias, des bouleversements qui affectent l'individu hypermoderne que des nouvelles modalités de 
concevoir le lien démocratique? Bref, le pamphlet, loin de s'être éteint, pourrait continuer sous des formes nouvelles, en des lieux inédits, à souffler les braises de la parole incendiaire.

\section{L'énonciation ordinaire du blâme}

Ce nouvel espace agonique semble se caractériser par un processus de "démocratisation» du pouvoir de blâmer. L'activité vitupérative, si longtemps portée par la figure du parrèsiaste et ses nombreux avatars historiques dont le pamphlétaire serait le dernier en date, connait actuellement dans les régimes démocratiques une reconfiguration profonde qui touche non seulement les qualités du locuteur, mais aussi les propriétés mêmes de la critique et probablement le sens accordé au dire-vrai. Depuis plusieurs années, il est fait état d'une crise de la démocratie représentative. De nombreux symptômes viennent illustrer cette thèse : abstention structurelle forte, érosion des militantismes partisans et syndicaux, défiance à l'encontre des autorités sociales et politiques traditionnelles, montée des radicalités, etc. Les observateurs constatent également un renouvellement des formes de participation politique et d'engagement, des demandes en faveur d'une plus grande transparence des décisions publiques. Le citoyen entend désormais se montrer plus vigilant, en complétant son rôle d'électeur par un ensemble de pratiques de surveillance, d'empêchement et de correction (Rosanvallon, 2006). Les conséquences de ces mutations ont été longuement analysées tant au niveau de l'art de gouverner que des redéfinitions de la citoyenneté démocratique. Mais peu d'études ont prolongé l'enquête, en s'interrogeant sur ce qui avait changé concernant l'espace public de la critique. Deux éléments peuvent être versés au dossier.

Tout d'abord, l'espace de la vitupération ne se réduit plus aujourd'hui à la presse, au livre et aux différents tréteaux du discours oral. La nouvelle géographie du blâme se situe désormais sur la toile, dans le foisonnement des blogs, des chats et des forums. Protégée par l'anonymat des pseudos, une parole décomplexée s'affranchit de certaines des contraintes de la discursivité classique (orthographe, style, argumentation) pour inaugurer, semble-t-il, un mode contemporain de la critique. L'outil Internet offre en effet de nouvelles opportunités pour publiciser la vitupération, la faire circuler, telle une nouvelle monnaie d'échange. L'interactivité des sites accroit l'impression de dialogue et surtout favorise une logique de surenchère, une montée en invective. La nouvelle topographie de la vitupération combine donc la totale dissimulation de l'énonciateur et l'extrême publicité de l'énoncé, redessinant ainsi les contours d'un pouvoir de tout dire dont la seule responsabilité réside désormais dans les limites imposées par le modérateur. Dès lors, la notion de risque, si consubstantielle à la notion de parrêsia, disparait, ou se reporte juridiquement sur l'opérateur 
du site. Ce déplacement de la scène vitupérative modifie donc l'essence même du pouvoir de tout dire. Ce dernier demeure plus que jamais recherché par les acteurs médiatiques, mais il est désassorti de toute prise de risque. A contrario, on peut se demander si de nouvelles formes de militantisme et de construction de la critique, pour l'heure encore minoritaires et qui requièrent l'anonymat, l'invisibilité, et surtout la double démythification du héros militant et de la parole publique, ne renouent pas d'une certaine manière avec l'expérience de la parrhésie, en jouant à la fois la prise de risque et la parole franche (sur le modèle de $L^{\prime}$ 'insurrection qui vient) ${ }^{1}$.

Un second élément doit être pris en considération dans la réévaluation actuelle de l'espace agonique : la consécration de l'individu ordinaire dont le témoignage aujourd'hui valorisé par les médias, les professionnels de la politique, mais aussi les sociologues, devient une vérité en soi, censée justement tout dire, en court-circuitant l'analyse et la démonstration. Un nouvel impératif de réaction traverse désormais l'économie des discours sociaux. Les journaux d'information radiophoniques, les articles de journaux mis en ligne font tous un appel à «réagir» à l'actualité, à l'article, c'est-à-dire à ne pas commenter, mais à formuler une réponse courte, fondée sur le ressenti, l'affectivité primaire, ouverte au jeu de mots, à l'insulte, à l'amalgame. Des auditeurs ou internautes, que l'idéal médiatique de la convivialité réduit à des prénoms et des tutoiements, deviennent les nouvelles figures de la critique vitupérative dans ces arènes «mosaïques» (François, Neveu, 1999). Le jeu du témoignage réactif exige des énoncés pathétiques, violents et sans nuance. Tout dire sur tout, sans préjuger de sa compétence à dire, telle pourrait être l'espace d'une nouvelle opinion publique, plus authentique car d'origine plus ordinaire. On disait hier populaire. La vitupération serait donc démocratisée au sens où l'énonciation de la critique semble désormais ne requérir ni figure héroïque, ni prise de risque. Le blâme public serait devenu, grâce aux nouveaux outils de communication, l'expression d'une citoyenneté directe et réactive. Le pouvoir de tout dire serait aujourd'hui délogé des porte-parole pour se voir réapproprié par les gens ordinaires. La parole vitupérative, comme syndrome de l'immédiateté, ne supporterait plus aucune représentation, aucune autre incarnation que celle de la figure du citoyen banal et inquisiteur. Tel est aujourd'hui le récit enchanté tenu par certains médias et sociologues naïfs qui imaginent que l'homme sans titre accéderait désormais aisément à l'espace de la parole publique.

\section{La polyphonie des vérités}

Le nouvel âge de la vitupération, ainsi remodelé par les mutations du champ médiatique et des règles du jeu démocratique, doit également tenir compte

1. Comité invisible, 2007, L'insurrection qui vient, Paris, La Fabrique. 
d'un autre facteur, autrement plus problématique, que l'on pourrait appeler le statut actuel de la vérité. Souvenons-nous en effet que c'est au nom d'une vérité, souvent incandescente, que se réalisait le contrat de la parrêsia. La question n'étant pas bien entendu de chercher à objectiver cette vérité, mais de la considérer comme une sorte de voix intérieure et impérieuse qui conduit l'énonciateur à engager sa personne dans une logique du risque. L'individu hypermoderne, dont les sociologues ont montré les métamorphoses de l'identité contemporaine, vit dans des sociétés qui connaissent un brouillage des repères, une tendance à l'effacement des frontières entre intime, privé et public. Dans ces conditions générales de vulnérabilité accrue, l'une de ses principales caractéristiques consiste en un besoin inassouvi de reconnaissance, qui prendra différentes formes : renforcement des ancrages identitaires, revendication d'authenticité, droit à la différence (Le Bart, 2008). Ces demandes de singularité affectent l'opération même de véridiction. Elles accroissent les sentiments de suspicion à l'encontre des vérités présentées comme incontestables ou officielles, et conduisent à des expressions de plus en plus élaborées de relativisme et de mise en perspective des vérités. La vérité n'est plus portée par un Grand Récit militant, historique ou religieux, elle énonce un point de vue personnel et entre donc désormais dans le monde du discutable.

Cela est certes aujourd'hui bien connu, mais ce qui nous intéresse ici concerne les effets de cette polyphonie des vérités sur l'expérience de la critique vitupérative. Ou pour le dire autrement, comment se forge l'opération du dire vrai dans un tel régime de véridiction? Le marché des vérités ne risque-t-il pas de diluer la performance critique de la parrhésie? Le pamphlétaire de la seconde moitié du $19^{e}$ siècle évoluait dans un champ limité et sa parole singulière pouvait plus aisément faire entendre sa vérité. Position institutionnelle, efficacité rhétorique le rendaient audible, mieux, identifiable. "Ça c'est du Rochefort! » disait-on alors. Il est désormais beaucoup plus difficile dans l'espace public de la critique de mobiliser un pouvoir de tout dire autour d'une vérité socialement considérée comme indiscutable. La tangibilité des valeurs diffuse une sorte de doute épistémologique sur ce genre de prétention. Les seules vérités susceptibles de recueillir des soutiens unanimes aujourd'hui renvoient à la manière dont nos sociétés construisent les frontières de leur espace moral et réinventent les figures de l'intolérable : l'enfant maltraité, la victime, etc. (Fassin et Bourdelais, 2005). En dehors de ce périmètre de la sacralité, la prise de parole au nom d'une vérité, la sienne, celle de son groupe de référence, est à la fois de plus en plus accessible aux individus et de moins en moins susceptible de se voir accréditer une légitimité plus élargie. Le pamphlet hier défendait une vérité objective à la fois sociale et morale; aujourd'hui, le nouvel espace de la vitupération délivre une vérité subjective, une sorte de voix intérieure irrépressible qui démonétise la notion même de 
vérité. La vitupération se diffracte en micro-réactions acrimonieuses, en actes de vandalisme verbal, où le tout dire sur tout ressemble davantage à une invitation à ne rien faire. Comme si la liberté de ton inhibait tout pouvoir d'agir.

Nous sommes parti d'un modèle matriciel d'énonciation de la critique. Expression première, mais profondément inscrite dans la configuration sociohistorique qui la vit naitre, la parrêsia fonctionne comme la mémoire d'un genre vitupératif en constante recomposition. II n'y a en effet pas d'essence de la parole qui blâme et qui dénonce, mais une généalogie de modalités capables à la fois de partager des mêmes airs de famille et de s'inscrire dans des processus de mutation. Le pamphlet est donc l'une de ces expressions historiques et localisées. Nous avons surtout essayé de montrer comment aujourd'hui l'esprit d'agôn peut à la fois participer des «arts de la résistance» à la domination (Scott, 1990), et d'une relative démocratisation du débat public où celui qui prétend désormais pouvoir tout dire, revendique en réalité qu'aucune parole n'a de valeur, et que l'interdit relève de son seul jugement (Descombes, 1977). L'émergence contemporaine du «vitupérateur sans qualité » accrédite donc l'idée d'un nouvel impératif critique et plaintif comme expression de démocratie directe. Elle fait peut-être partie de ces différentes expériences actuelles de réappropriation par les citoyens de la démocratie, au sein desquelles figurent des espaces critiques alternatifs dotés d'une parole plus politisée donc plus risquée, mais il nous semble qu'elle ne répond pour l'instant qu'imparfaitement au souci éthique du dire-vrai que portait la parrêsia des anciens Grecs.

\section{Références}

ACHARD Guy, 1981, Pratique rhétorique et idéologie politique dans les discours «optimates » de Cicéron, Leyde, Brill.

Agamben Giorgio, 2005, Profanations, Paris, Rivages.

ANGENOT Marc, 1997, La propagande socialiste, Montréal, L'Univers du discours.

- 2005 [1982], La parole pamphlétaire. Typologie des discours modernes, Paris, Payot, Rivages.

- 2008, Dialogues de sourds, Paris, Mille et une nuits.

BARrows Susanna, 1990, Miroirs déformants. Réflexions sur la foule en France à la fin duxixe siècle, Paris, Aubier.

Berrendonner Alain, 1981, Éléments de pragmatique linguistique, Paris, Minuit.

BoltANSKI Luc, 1985, "La dénonciation", Actes de la recherche en sciences sociales, $\mathrm{n}^{\circ} 1, \mathrm{p} .3-40$.

BoltANSKI Luc, THÉvenot Laurent, 1991, De la justification. Les économies de la grandeur, Paris, Gallimard.

BOuCHET Thierry éd., 2005, L'insulte (en) politique, Dijon, Éditions universitaires de Dijon. 
ChARTIER Pierre, 2005, Théorie du persiflage, Paris, PUF.

Chauvaud François éd., 2008, La haine. Histoire et actualité, Rennes, Presses universitaires de Rennes.

DELEPLACE Marc éd., 2009, Les discours de la haine. Récits et figures de la passion dans la Cité, Lille, Presses universitaires du Septentrion.

Descombes Vincent, 1977, L'inconscient malgré lui, Paris, Minuit.

Dupont Florence, Valette-Cagnac Emmanuelle, 2004, Façons de parler grec à Rome, Paris, Belin.

FASSIN Didier, BourdelaIs Patrice éd., 2005, Les constructions de l'intolérable, Paris, La Découverte.

Foucault Michel, 2001, L'herméneutique du sujet. Cours au Collège de France, 19811982, Paris, Gallimard, Le Seuil.

- 2008, Le gouvernement de soi et des autres. Cours au Collège de France, 1982-1983, Paris, Gallimard, Le Seuil.

- 2009, Le courage de la vérité. Cours au Collège de France, 1984, Paris, Gallimard, Le Seuil.

FrançoIs Bastien, NeVeu Érik éd., 1999, Espaces publics mosaïques. Actions, arènes et rhétoriques des débats publics contemporains, Rennes, Presses universitaires de Rennes.

HEINICH Nathalie, 1991, La gloire de Van Gogh. Essai d'anthropologie de l'admiration, Paris, Minuit.

JoAnna Jean, 1999, Pratiques politiques des députés français au xıxe siècle. Du dilettante au spécialiste, Paris, L'Harmattan.

Jounaud Christian, 1985, Mazarinades. La Fronde des mots, Paris, Aubier.

- 2000, Les pouvoirs de la littérature, Paris, Gallimard.

KoseleECK Reinhart, 1979, Le règne de la critique, Paris, Minuit.

Le BART Christian, 2008, L'individualisation, Paris, Les Presses de Sciences Po.

ORWELL George, 1995, Essais, articles, lettres (1940-1943), t. II, Paris, Ivréa.

OSt François, 2005, Sade et la loi, Paris, Odile Jacob.

Rosanvallon Pierre, 2006, La contre-démocratie. La politique à l'âge de la défiance, Paris, Le Seuil.

- 2008, La légitimité politique. Impartialité, réflexivité, proximité, Paris, Le Seuil.

Roussın Philippe, 2005, Misère de la littérature, terreur de l'histoire, Paris, Gallimard.

Scotr James, 1990, Domination and the Art of Resistance, New Haven, Londres, Yale University Press.

SLoterdijk Peter, 2007, Colère et temps, Paris, Libella / Maren Sell.

THÉRENTY Marie-Ève, 2007, La littérature au quotidien, Paris, Le Seuil.

Tilly Charles, 2008, Credit and Blame, Princeton, Londres, Princeton University Press.

WALzer Michael, 1996, La critique sociale au xxe siècle, Paris, Métailié. 